\title{
Nuclear density functional theory with a semi-contact 3-body interaction
}

\author{
Denis Lacroix ${ }^{1, a}$ and Karim Bennaceur $2,3, b$ \\ ${ }^{1}$ Institut de Physique Nucléaire, IN2P3-CNRS, Université Paris-Sud, F-91406 Orsay Cedex, France \\ ${ }^{2}$ Université de Lyon, F-69003 Lyon, France; Institut de Physique Nucléaire de Lyon, CNRS/IN2P3, Université Lyon 1, F-69622 \\ Villeurbanne Cedex, France \\ ${ }^{3}$ Department of Physics, PO Box 35 (YFL), Fl-40014 University of Jyväskylä, Finland
}

\begin{abstract}
Theories combining nuclear density functional approach (DFT) and effects beyond the independent particle/quasi-particle limit have attracted much attention recently. In particular, such theories, generically referred as "beyond mean-field" (BMF) seem unavoidable to account for both single-particle effects and complex quantum internal phenomena in nuclear finite many-body nuclear systems. It has been realized recently that BMF theories might lead to specific difficulties when applied within the nuclear DFT context. An example is the appearance of divergences in configuration mixing approaches. A short summary of the difficulties is given here. One source of problem is the use of energy functional of non-integer powers of the density. We show that such dependence can be mimicked by a suitable choice of a three-body interaction. Application on infinite nuclear matter in various spin-isospin channels will be given.
\end{abstract}

\section{Introduction}

BMF theories within nuclear DFT can be motivated by many-body techniques starting from a bare Hamiltonian. Most often, the starting point of the nuclear DFT, also called Energy Density Functional (EDF) theory is an effective interaction that is used to obtain the form of the functional. This guidance is very useful and has led to a framework allowing to treat nuclear structure, dynamics and thermodynamics. In recent years, efforts have been made to describe complex phenomena beyond the independent particle/quasi-particle picture. The inclusion of such phenomena have pointed out that theories such that perturbation theory $[1,2]$ or configuration mixing [3-6], in contrast to the pure Hamitonian case, should be used with specific care within the EDF framework.

In the present article, some difficulties associated to configuration mixing are illustrated. Among these difficulties, it turns out that terms written as $\rho^{\alpha}$ where $\alpha$ is not an integer can not be used without difficulty in combination with standard configuration mixing based on projections techniques. However, as will be discussed below, such density dependence of the functional often appears as a useful choice in many situations to have local terms in the density functionals. We show here that this density dependence can be appropriately mimicked using a recently proposed simplified three-body interaction [7].

\footnotetext{
a e-mail: lacroix@ipno.in2p3.fr

be-mail: bennaceur@ipnl.in2p3.fr
}

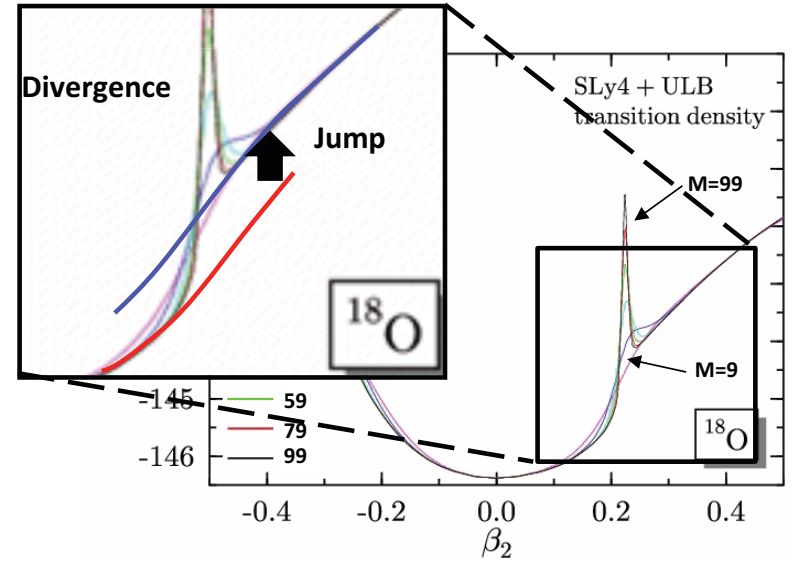

Figure 1. (Color online) Illustration of the difficulties encountered in the EDF approach when combined with configuration mixing. Here, an energy landscape of a nucleus is shown as a function of the quadrupole deformation parameter $\beta_{2}$. The energy is obtained by discretizing the energy landscape of the nucleus at various deformation and by performing configuration mixing. When the number of points $M$ used in the discretization increases, jumps or even divergences appears in the energy (adapted from [4-6]).

\section{The EDF and configuration mixing}

The nuclear EDF has strong similarities with the DFT approach used in condensed matter. In general, a trial state $\Psi$ (Slater determinant and/or Quasi-particle vacuum) is used to construct the normal density $\rho$ and pairing tensor $\kappa$ that are ultimately used in the energy density $\mathcal{E}(\rho, \kappa)$. This fi- 
nally gives the DFT scheme:

$$
\Psi \quad \longrightarrow \quad(\rho, \kappa) \longrightarrow \mathcal{E}(\rho, \kappa) .
$$

This scheme, that is often referred as the mean-field level of EDF, is very powerful to describe many facets of the atomic nucleus. However, it is mainly suited to access ground state properties and additional efforts is required to provide theoretical prediction for excited states.

Guided by the Hamiltonian case, the natural method to obtain spectroscopic information with many-body states having proper symmetries is to use configuration mixing techniques. Then, the single trial state is replaced by a new state $\Phi$ written as a coherent superposition of a set of trial states $\Psi(q)$

$$
\Phi=\int f(q) \Psi(q) d q .
$$

Here, $q$ is a generic notation for a set of collective coordinates that eventually allows for symmetry restoration like in the case of the particle number or the angular momentum projection. This strategy however leads to specific problems, one of them being illustrated in Fig. 1. A complete discussion of the problems showing up when combining EDF with BMF technique is out of the scope of the present article and a detailed discussion can be found in Refs. [4-6]. Let us just remind two important issues (i) density dependence with non-integer powers in the energy density cannot be used with configuration mixing in the general case (ii) most of the difficulties encountered in BMF theories do not exist if an Hamiltonian (i.e. without density dependent effective vertex) is used as a starting point for the EDF and no approximation is made to construct the functional especially regarding the Pauli principle and the Coulomb field. In particular, this has renewed the construction of EDF in the Local Density Approximation (LDA) from zero-range interaction including 3-body and/or 4-body terms [8,9]. However, as it is discussed below, it seems unclear if a LDA approximation of the EDF can properly describe Fermi liquids in various density regimes.

\section{Usefulness of non-integer density dependence in functional theories for Fermi liquids}

In Fig. 2, illustration of an equation of state (EOS) obtained using the Sly5 Skyrme functional for the neutron matter and symmetric nuclear matter are displayed as a function of density. The different black circles indicate cases where a functional containing terms $\rho^{\alpha}$ with noninteger values of $\alpha$ seems adequate. The most evident case is the low density limit in Fermi liquids, (1) in Fig. 2. In that case, for sufficiently dilute systems, the effect of the interaction becomes perturbative and the energy is given by the Lee-Yang formula [10] in terms of the Fermi momentum $k_{F}$ as:

$$
\frac{E}{A}=\frac{E_{0}}{A}\left(1+\frac{10}{9 \pi}\left(a k_{F}\right)+\frac{4}{21 \pi^{2}}(11-2 \ln 2)\left(a k_{F}\right)^{2}\right)
$$

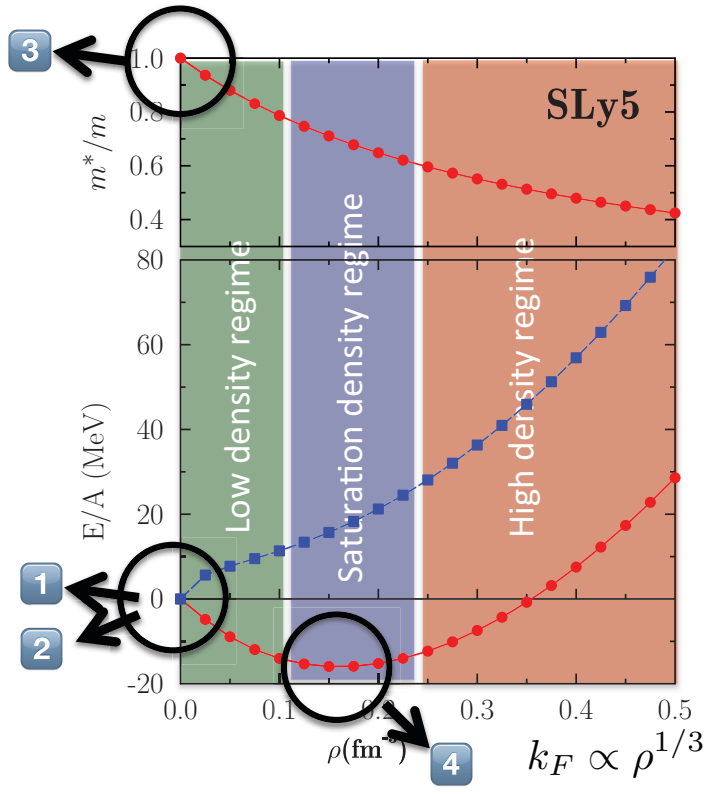

Figure 2. (Color online) Bottom: Equation of state in infinite matter obtained as a function of density using the Sly5 Skyrme functional in symmetric matter (red filled circles) and neutron matter (blue filled squares). Top: Associated effective mass. The different shaded area correspond to different density regimes. The different numbers (from 1 to 4 ) correspond to different places where a terms $\rho^{\alpha}$ with $\alpha$ non-integer is useful.

where $a$ is the scattering length of the interaction and $E_{0} / A=3 / 5\left(\hbar^{2} k_{F}^{2} /[2 m]\right)$ is the Free Fermi gas kinetic energy per particle. In this limit, the effective mass can also be expanded in $k_{F}$ through the Galitskii formula [10]:

$$
\left(\frac{m^{*}}{m}\right)=1+\frac{8}{15 \pi^{2}}(7 \ln 2-1)\left(k_{F} a\right)^{2} .
$$

Noting that $k_{F} \propto \rho^{1 / 3}$, we directly see that both the energy and the effective mass can be parametrized with terms $\rho^{\alpha}$ where $\alpha$ is not an integer. This directly stems from the fact that the natural expansion parameter at low density is the Fermi momentum and not directly the density itself.

Another case that has attracted much attention in the last decade is a Fermi liquid at low density and very large scattering (infinite) length, i.e. the unitary regime. This situation is close to the case of neutron matter (shown by (2) in Fig. 2). In the strict unitary regime, the energy directly becomes proportional to the free gas energy with $\frac{E}{A}=\xi \frac{E_{0}}{A}$. Here $\xi$ is a constant sometimes called Bertsch parameter. This very simple form has been used in Refs. [11, 12] (see also [13]) to suggest a rather minimalist DFT-LDA functional for unitary gas where the energy density writes

$$
\mathcal{E}[\rho, \kappa]=\alpha \frac{\tau(\mathbf{r})}{2}+\beta c \rho^{5 / 3}(\mathbf{r})+\gamma \frac{|\kappa(\mathbf{r})|^{2}}{\rho(\mathbf{r})^{1 / 3}} .
$$

Here, $\tau(\mathbf{r})$ is the kinetic density. This functional is simple thanks to the inclusion of non-integer density dependences both in the mean-field and pairing channels. 
The usefulness of such $\rho^{\alpha}$ terms is not restricted to the low density regime. Indeed, in the nuclear physics context, it was realized rapidly that a proper description of the incompressibility around saturation ((4) in Fig. 2) can only be achieved in the EDF-LDA approach, without increasing too much the complexity in the functional, by introducing a $\rho^{\alpha}$ term [14].

\section{Nuclear DFT starting from a Hamiltonian}

From the above discussion, we see that the use density dependent terms $\rho^{\alpha}$ allows for the construction of an EDF with a sufficiently simple expression when a LDA approximation is seek. However, having in mind BMF approximation, we have the dilemma that such term should be avoided and that it would be desirable to design the functional directly from an Hamiltonian.

At present, two strategies have been tested along this line:

- Keeping the LDA approach, and following the pioneering work of Skyrme, Hamiltonian based on zero-range interaction of increasing complexity have been considered $[8,9]$. The resulting EDF can then only depend on integer powers of the different spin/isospin densities. It is clear that an energy that writes as a polynomial of the density with a sufficient number of terms to adjust can eventually reproduce the effect of $\rho^{\alpha}$ at least around the saturation point. However, LDA based on zero range interaction cannot describe the low density regime.

- Alternatively, one can relax the LDA constraint and starts from finite-range many-body interaction. As we discussed in [7], if the Hamiltonian contains only twobody interaction, it can neither describe nuclear systems at low density nor at saturation density. At least a threebody interaction should be included. A first attempt in that direction was made in Ref. $[15,16]$ to get a functional starting from a finite range 3-body interaction. It was shown that a specific choice of the interaction can provide a proper description of the low density behavior.

In the following, we show that the introduction of a simplified 3-body interaction to obtain an EDF from a Hamiltonian can also be useful around saturation. Some technical details associated to this work can be found in Ref. [7].

\subsection{Semi-contact 3-body interaction}

While finite-range 2-body interaction, like the Gogny interaction, is rather standardly used nowadays, 3-body interaction remains challenging to implement in mean-field codes. For this reason, we recently introduced the concept of semi-contact 3-body interaction. We start from the Jacobi coordinates $\mathbf{r}_{i j}=\left(\mathbf{r}_{i}-\mathbf{r}_{j}\right), \mathbf{R}_{i j}^{k}=\mathbf{r}_{k}-\left(\mathbf{r}_{i}+\mathbf{r}_{j}\right) / 2$ where $\left(\mathbf{r}_{i}, \mathbf{r}_{j}, \mathbf{r}_{k}\right)$ are the coordinates of three particles, $(i, j, k)$. We called semi-contact 3-body interaction, an interaction that is non-zero only if $\mathbf{R}_{i j}^{k}=0$. The starting semi-contact 3body interaction is written as: $\bar{v}_{i j k}=\left(v_{i j k}+v_{i k j}+v_{k i j}\right) / 3$
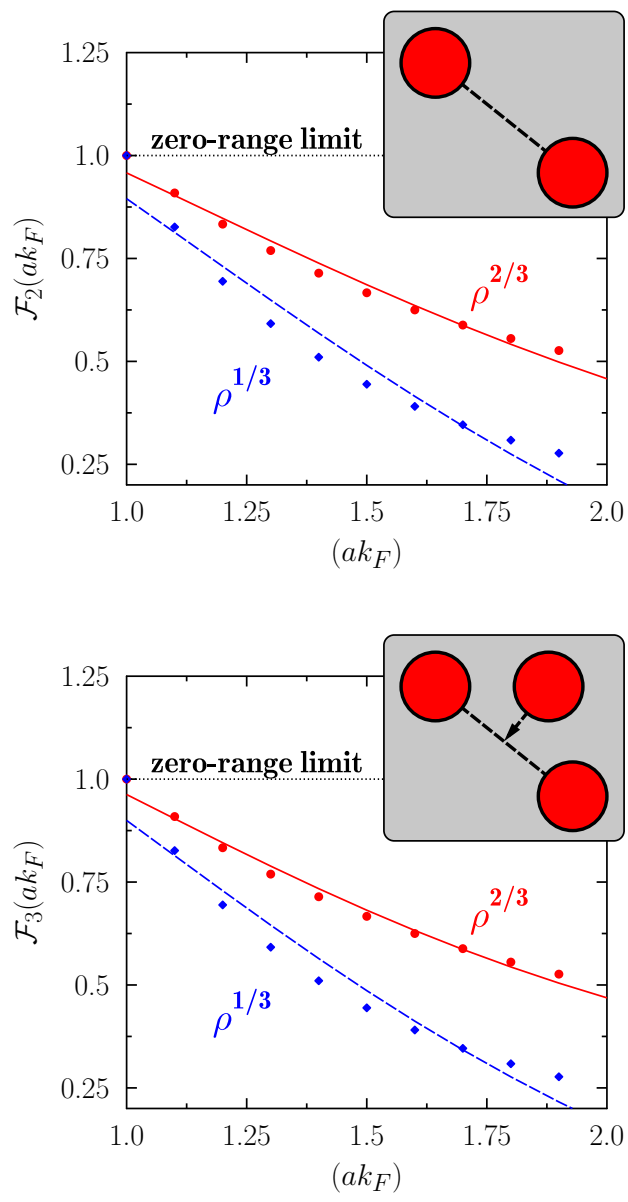

Figure 3. (Color online) $\mathcal{F}_{3}\left(k_{F} a\right)$ as a function of $\left(a k_{F}\right)$ obtained by adjusting the interaction parameters to get a dependence similar to $1 /\left(a k_{F}\right)[\alpha=2 / 3]$ (red solid line) or $1 /\left(a k_{F}\right)^{2}[\alpha=1 / 3]$ (blue dashed line). The reference curves $1 /\left(a k_{F}\right)$ (red filled circles) and $1 /\left(a k_{F}\right)^{2}$ (blue filled diamonds) are also shown. Note that in nuclear physics $\left(a k_{F}\right) \simeq 1.5$ at saturation.

with (see [7] for more details)

$$
v_{i j k}=v_{i j} \delta\left(\mathbf{r}_{k}-\left[\frac{\mathbf{r}_{i}+\mathbf{r}_{j}}{2}\right]\right),
$$

and

$$
v_{i j}=\left\{V_{0}(r)+V_{\sigma}(r) P_{\sigma}+V_{\tau}(r) P_{\tau}+V_{\sigma \tau}(r) P_{\sigma} P_{\tau}\right\} .
$$

Here, $\sigma$ and $\tau$ denotes the different spin and isospin channels of the interaction. The later expression is the standard form of a finite-range 2-body interaction decomposed in different spin/isospin channels. For simplicity, the interaction was assumed to have the same form factor in all channel $V_{\alpha}(r)=v_{\alpha} g(r)$ where $g(r)$ is a Gaussian function while $v_{0}, v_{\sigma}, v_{\tau}$ and $v_{\sigma \tau}$ are independent interaction strength parameters. The presence of a $\delta$ function in Eq. (5) simplifies some technical aspects compared to general 3-body forces. Starting from Eq. (5), the energy per particle can be written as a functional of $\left(a k_{F}\right)$ as

$$
\frac{E_{3 b}}{A}=\frac{\rho^{2}}{6} \mathcal{F}_{3}\left(a k_{F}\right),
$$



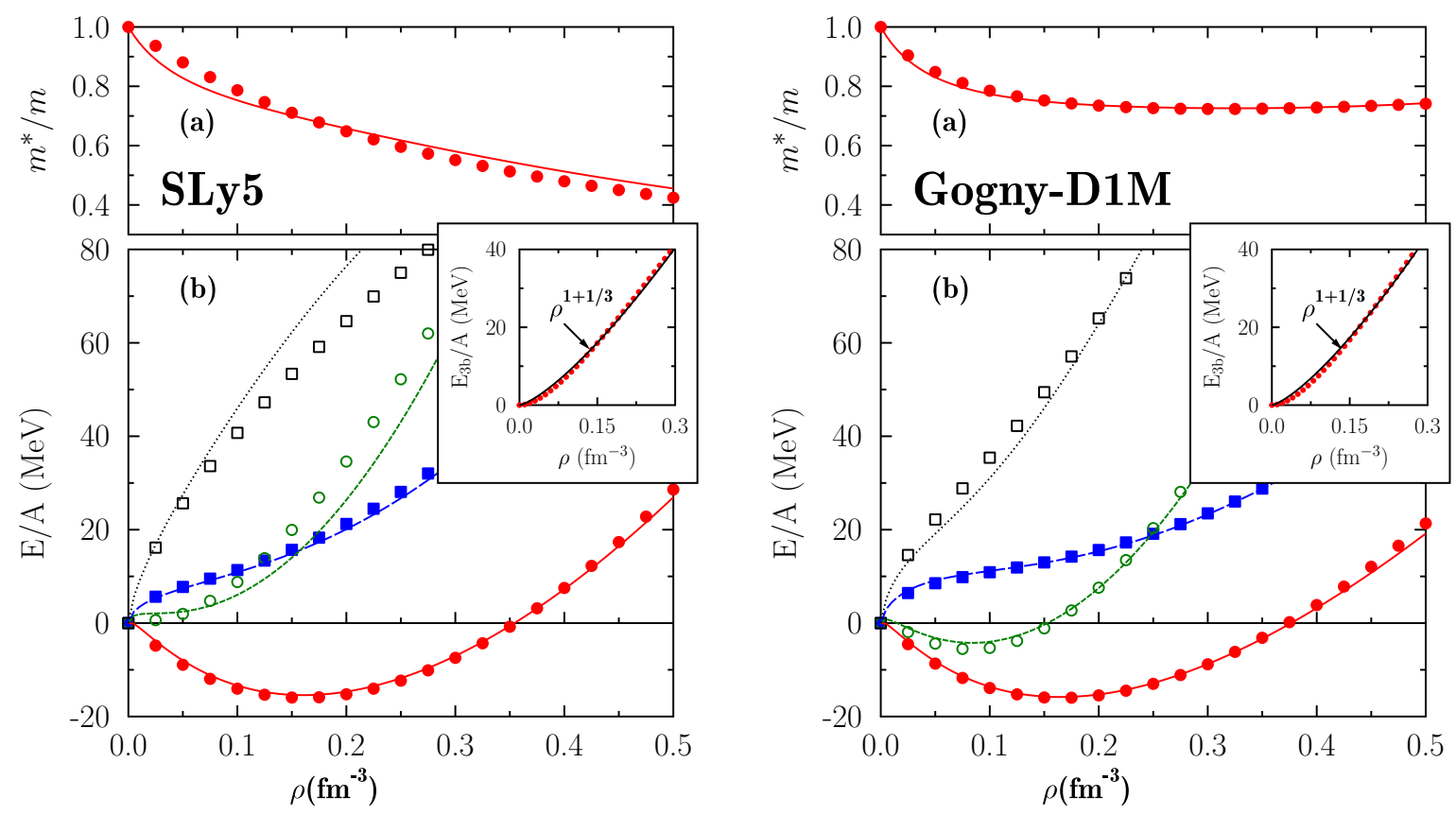

Figure 4. (Color online) Scalar-Isoscalar effective mass (a) and equations of state (b) of symmetric matter (red), neutron matter (blue), polarized matter (green) and polarized neutron matter (black) as a function of density. Left: the SLy5 functional (markers) is used as the reference functional. The SLy5 ${ }^{3 \mathrm{~b}}$ including the [2-body] + 3-body functional are shown with lines. Right: Same for the D1M as reference functional and $\mathrm{D}^{3} \mathrm{M}^{3 \mathrm{~b}}$ as the new functional. In both cases, in the insets, the contribution of the 3-body functional to the energy per particle in symmetric matter is represented. A simple function proportional to $\rho^{1+\alpha}$ has been adjusted on it with $\alpha \simeq 1 / 3$ for an optimal fitting the vicinity of saturation density.

where $a$ now stands for the range of the Gaussian interaction. For comparison, it is also interesting to note that the energy of a two-body interaction given by Eq. (6) writes as:

$$
\frac{E_{2 b}}{A}=\frac{\rho}{2} \mathcal{F}_{2}\left(a k_{F}\right)
$$

The explicit form of the two functionals $\mathcal{F}_{2}\left(a k_{F}\right)$ and $\mathcal{F}_{3}\left(a k_{F}\right)$ depends on the spin and isospin content of nuclear matter. Their expressions for symmetric, neutron matter, polarized and neutron polarized matter are explicitly given in Ref. [7]. In the limit of zero-range interaction, i.e. $a \rightarrow 0$, one recovers the standard LDA functional form with

$$
\frac{E_{3 b}}{A} \propto \rho^{2}, \text { and } \frac{E_{2 b}}{A} \propto \rho .
$$

When a finite-range interaction is used, a richer density dependence of the energy can be obtained. As an illustration, we show in Fig. 3 that a proper adjustment of the interaction parameters can lead to a dependence equivalent to $\rho^{\alpha}$ with $\alpha=1 / 3$ or $\alpha=2 / 3$ in a density region around saturation. These values of $\alpha$ corresponds to values that are standardly used in Skyrme EDF or Gogny EDF to properly describe compressibility property of symmetric nuclear matter at saturation.

\subsection{Skyrme or Gogny EDF without density dependent term}

Let us now return to the original goal that is (i) to obtain the EDF form from a Hamiltonian (ii) to avoid density dependent terms with non-integer powers of the density. As we have seen above, the semi-contact 3-body interaction can appropriately replace the term $\rho^{\alpha}$ in the functional. Therefore, we have considered either the Skyrme functional or the Gogny functional where the density dependent term is replaced by the 3-body functional (8). To demonstrate that the new functional can be competitively compared to other EDFs, we have used the original EOS in different spin/isospin channels given by standard Skyrme or Gogny interactions that include the term $\rho^{\alpha}$ as reference EOSs. Then we have used these reference EOSs to adjust parameters of the new functional to get comparable results. In the present work, we used the SLy5 [14] and D1M [17] respectively for the reference EOSs using Skyrme or Gogny interactions. The new functionals are called SLy5 ${ }^{3 b}$ and $D 1 M^{3 b}$ respectively. The results given by the parameters fits are shown in Figs. 4. It is worth mentioning that all parameters of the functional have to be adjusted simultaneously to get a proper reproduction of the reference EOSs in all channels.

The new functionals SLy5 ${ }^{3 \mathrm{~b}}$ and $\mathrm{D}^{3} \mathrm{M}^{3 \mathrm{~b}}$, where the density dependent term is replaced by the semi-contact 3body interaction, provide with a very good reproduction of the saturation properties of the reference functionals, SLy5 
and D1M, for all considered cases. In particular, the compression modulus that was one of the motivations for the introduction of the $\rho^{\alpha}$ term in the Skyrme and Gogny interactions has a reasonable value (see Table 1) close to the empirical ones.

Table 1. Values of the saturation density $\rho_{\text {sat }}$, binding energy $E / A$, compression modulus $K_{\infty}$ and isoscalar effective mass $\mathrm{m}^{*} / \mathrm{m}$ for the functionals considered in this work.

\begin{tabular}{|l|c|c||c|c|}
\cline { 2 - 5 } \multicolumn{1}{c|}{} & SLy5 & SLy5 $^{3 \mathrm{~b}}$ & D1M & D1M $^{3 \mathrm{~b}}$ \\
\hline$\rho_{\text {sat }}\left[\mathrm{fm}^{-3}\right]$ & 0.160 & 0.161 & 0.165 & 0.165 \\
\hline$B / A[\mathrm{MeV}]$ & -15.98 & -15.42 & -16.02 & -15.82 \\
\hline$K_{\infty}[\mathrm{MeV}]$ & 229.92 & 236.59 & 224.98 & 228.58 \\
\hline$m^{*} / m$ & 0.697 & 0.691 & 0.746 & 0.744 \\
\hline
\end{tabular}

It is finally worth to mention that not only the energy is well reproduced but also the density dependence of the effective mass, shown in the panels (a) of Fig. 4.

\section{Conclusion}

In recent years, important efforts have been made to apply the EDF approach including Beyond Mean-Field effects. It turned out that some functionals that were very successful at the mean-field levels need to be improved to lead to safe calculations in the BMF sector. In particular a density dependent term $\rho^{\alpha}$ induces serious problems in configuration mixing theories. Here we show that this term can be accurately replaced by a simplified 3-body interaction that serves to obtain the energy density functional. First applications to infinite nuclear matter shows that it can properly describe saturation properties like binding energy, incompressibility and/or effective mass. The use of finite range interaction, especially 3-body finite range interactions, is more demanding that zero-range interaction. Work is in progress to apply the new functional to finite systems including pairing effects.

\section{Acknowledgements}

We thank M. Bender, J. Dobaczewski, T. Duguet and M. Kortelainen for discussions. This work has been supported in part by the Academy of Finland and University of Jyväskylä within the FIDIPRO programme.

\section{References}

[1] K. Moghrabi, M. Grasso, G. Colò, and N. Van Giai Phys. Rev. Lett. 105, 262501 (2010)

[2] K. Moghrabi, M. Grasso, X. Roca-Maza, and G. Colò, Phys. Rev. C 85, 044323 (2012)

[3] J. Dobaczewski, M.V. Stoitsov, W. Nazarewicz, and P.-G. Reinhard, Phys. Rev. C 76, 054315 (2007)

[4] D. Lacroix, T. Duguet, and M. Bender, Phys. Rev. C 79, 044318 (2009)

[5] M. Bender, T. Duguet, and D. Lacroix, Phys. Rev. C 79, 044319 (2009)

[6] T. Duguet, M. Bender, K. Bennaceur, D. Lacroix, and T. Lesinski, Phys. Rev. C 79, 044320 (2009)

[7] Denis Lacroix and K. Bennaceur, Phys. Rev. C 91, 011302(R) (2015)

[8] K. Washiyama, K. Bennaceur, B. Avez, M. Bender, P.-H. Heenen, V. Hellemans, Phys. Rev. C 86, 054309 (2012)

[9] J. Sadoudi, T. Duguet, J. Meyer, and M. Bender, Phys. Rev. C 88, 064326 (2013)

[10] A.L. Fetter and J.D. Walecka, Quantum Theory of Many-Particle Systems (McGraw-Hill, New York, 1971)

[11] A. Bulgac, Phys. Rev. A 76, 040502R (2007)

[12] A. Bulgac, M.M. Forbes, and P. Magierski, The Unitary Fermi Gas: From Monte Carlo to Density Functionals, Lecture Notes in Physics Vol. 836 (SpringerVerlag, Berlin, Heidelberg, 2012), Chap. 9, pp. 305373.

[13] Y. Yu and A. Bulgac, Phys. Rev. Lett. 90, 222501 (2003); Phys. Rev. Lett. 90, 161101 (2003)

[14] E. Chabanat, P. Bonche, P. Haensel, J. Meyer, and R. Schaeffer, Nucl. Phys. A 635, 231 (1998)

[15] A. Gezerlis and G.F. Bertsch, Phys. Rev. Lett. 105, 212501 (2010)

[16] A. Gezerlis and G.F. Bertsch, Phys. Rev. C 85, 037303 (2013)

[17] F. Chappert, M. Girod, and S. Hilaire, Phys. Lett. B 668, 420 (2008) 